\title{
Atlas-Based Linear Volume-of-Interest (ABL-VOI) Image Correction
}

\author{
A. K. Maier ${ }^{1}$, Z. Jiang 2 , J. Jordan², C. Riess ${ }^{2}$, H. G. Hofmann' ${ }^{2}$, J. Hornegger ${ }^{2}$ \\ ${ }^{1}$ Siemens AG, Healthcare Sector, Forchheim, Germany \\ ${ }^{2}$ Pattern Recognition Lab, Friedrich-Alexander-University, Erlangen-Nuremberg, Germany
}

\begin{abstract}
Volume-of-interest imaging offers the possibility to image small volumes at a fraction of the dose of a full scan. Reconstruction methods that do not involve prior knowledge are able to recover almost artifact free images. Although the images appear correct, they often suffer from the problem that low-frequency information that would be included in a full scan is missing. This can often be observed as a scaling error of the reconstructed object densities. As this error is dependent on the object and the truncation in the respective scan, only algorithms that have the correct information about the extent of the object are able to reconstruct the density values correctly.

In this paper, we investigate a method to recover the lost low-frequency information. We assume that the correct scaling can be modeled by a linear transformation of the object densities. In order to determine the correct scaling, we employ an atlas of correctly scaled volumes. From the atlas and the given reconstruction volume, we extract patch-based features that are matched against each other. Doing so, we get correspondences between the atlas images and the reconstruction VOI that allow the estimation of the linear transform.
\end{abstract}

We investigated several scenarios for the method: In closed condition, we assumed that a prior scan of the patient was already available. In the open condition test, we excluded the respective patient's data from the matching process. The original offset between the full view and the truncated data was $133 \mathrm{HU}$ on average in the six data sets. The average noise in the reconstructions was $140 \mathrm{HU}$. In the closed condition, we were able to estimate this scaling up to $9 \mathrm{HU}$ and in open condition, we still could estimate the offset up to $23 \mathrm{HU}$.

\section{INTRODUCTION}

In the recent years the use of small implanted devices in neurological angiographic interventions has increased [1]. For the treatment of aneurysms, for example, flow diverters and pipeline stents are on the rise. While they are easy to deploy, they are barely visible in a fluoroscopic view. Using $3 \mathrm{D}$ reconstruction technologies, flow diverters can be visualized at a good image quality, as 3D reconstructions allow for low to medium contrast imaging. In terms of dose, however, 3D imaging protocols often impose a higher burden on the patient [2]. Thus, there is a new demand for low-dose 3D imaging of small devices.

Volume-of-interest (VOI) imaging offers a possible solution to this problem, as scanning of smaller volumes also reduces the dose delivered to the patient. However, this also imposes the constraint that the scan of the object of interest is incomplete with respect to its spatial extent. In reconstruction theory, this problem is known as the so-called interior problem. For an exact solution, either the extent of the object has to be known from a prior scan [3], or at least a tiny part of the object has to be known a priori [4].

In cases where no prior knowledge is available, the problem can also be solved by using heuristics. The better the heuristic is fulfilled, the better the outcome of these methods. These methods are often called truncation correction in the literature. Some of these are based on the extrapolation of the field-of-view that was not measured [5] [6] [7], while others extrapolate the view only implicitly [1][8]. Truncation correction approaches deliver a satisfying image quality; the solution however, is only approximate. Correct reconstruction is only guaranteed if the heuristic matches the imaged object exactly. If the heuristic assumption is violated, the reconstructions still suffer from low frequency artifacts. Figure 1 displays a difference image between a full scan reconstruction and a reconstruction based on a heuristic method comparable to [6]. The main difference in this case is an offset of about $150 \mathrm{HU}$. Furthermore, there is a slight intensity increase in the VOI reconstruction towards the boundary of the field-of-view. This kind of residual artifact is typical for most heuristic truncation correction methods. 


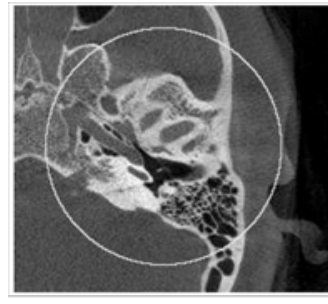

Full View Reconstruction

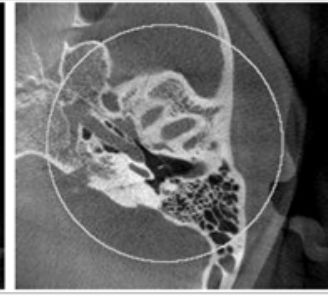

Truncation Corrected Reconstruction

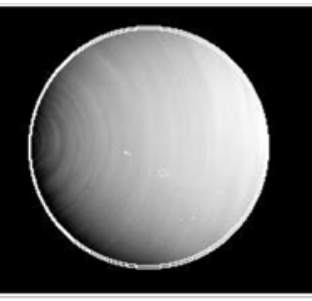

Difference Image
Figure 1. Comparison of a full view reconstruction with a truncation corrected reconstruction after [6]. The main difference between both consists of low-frequencies. Most of the difference comes from an offset.
In an interventional setting, this difference is often negligible as the imaged objects are medium to high contrast. In most cases, the physician wants to see high contrast details at high spatial resolution (as the bony structures in Figure 1). A correct scaling of the $\mathrm{HU}$ values in the reconstruction is still desirable, as they allow conclusions on the type of material and may be of further diagnostic interest. In addition, some correction steps that are applied in image domain rely on a correct scaling, e.g. noise reduction and ring correction algorithms [9].

In this paper, we estimate the missing offset of truncation correction methods by using image retrieval methods. In this way, we are able to include prior knowledge into the reconstruction process without having to rely on information of a specific patient. We detect patchbased features and search for similar features in a database. Then we use the matched patches to estimate the correct scaling of the images. In order to limit the amount of prior information that is included in this process, we only allow a global linear scaling to correct the image.

\section{MATERIAL \& METHODS}

3D image reconstruction was performed on a $512 \times 512 \times 512$ grid with different voxel sizes depending on the size of the VOI. All reconstructions were generated from short scans over an angular range of 200 degrees with 496 C-arm cone-beam projections with $1240 \times 960$ pixels at an isotropic resolution of $0.3 \mathrm{~mm}$ [10][11]. We used a sharp Shepp-Logan kernel in the filtering in order to preserve details at a high spatial resolution. The reconstructions of the full field-of-view covered a cylinder with a diameter of $25 \mathrm{~cm}$. In order to generate VOI scans the data was virtually collimated prior to the reconstruction. Truncation correction was performed with a method similar to [6].

Patch-matching was based on the Speed-up Robust Features (SURF [12]). We used an interest point detector based on the determinant of the Hessian in a scale space that covered three octaves, i.e. a magnification of up to $2^{3}$. Three octaves were deemed as sufficient for the 3D imaging capabilities of our angiographic C-arm system as the smallest considered voxel size was $0.1 \mathrm{~mm}$ the largest $0.5 \mathrm{~mm}$. For each interest point, a scale and rotation invariant 64 dimensional feature vector, as described in the original paper, was obtained. In order to augment the vector with reconstruction intensity values, we added additional 16 values that described a $4 \times 4$ patch around the detected point in the same orientation and resolution. In this manner, 80 dimensional feature vectors were obtained. Note that only the first 64 dimensions were used for the patch matching. In this manner, we do not have to actually retrieve the original images from the database. Only the 16 entries that describe the actual density values are used to estimate the correction transformation.

In order to correct the reconstructed intensities, we estimated a linear transform:

$$
f_{\text {corr }}(x, y, z)=a \cdot f(x, y, z)+b
$$

In this notation, $f(x, y, z)$ denotes the truncation corrected reconstruction, $f_{\text {corr }}(x, y, z)$ the image after correction, and $a$ and $b$ the parameters of the linear correction. To estimate reference correction parameters, we used the full field-of-view scan reconstructions and matching VOI reconstructions. Note that due to the high amount of noise, the parameters were not estimated with a least-square method [13], but a robust estimator using the random sample consensus (RANSAC [14] [15][16]). The same estimation method was also applied to estimate the correction transform from the detected correspondences. In contrast to the full field-of-view case, only the 16 values from each of the matched feature vectors that describe the reconstructed densities were considered for this estimation process. 
In total skull scans of six patients were investigated for first experimental results. Due to the limited size of the database, we investigated two scenarios: A best-case scenario in closed condition that included the tested patient's scan in the database and a worst-case scenario that included only the other five patients' data in the database. We consider the results of the best-case scenario as a kind of upper-bound for our method, while the worst case-scenario gives an intuition on the robustness of the method when only little training data is available. Note that the assumption that the database contains only head scans is not much of a limitation, as coordinate systems in the scanner may be used to locate the part of the body for database selection.

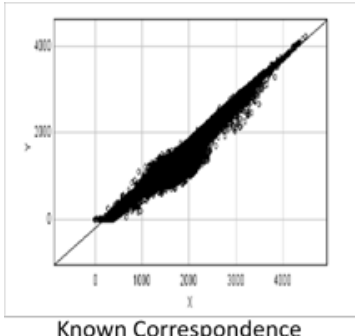

Known Correspondence

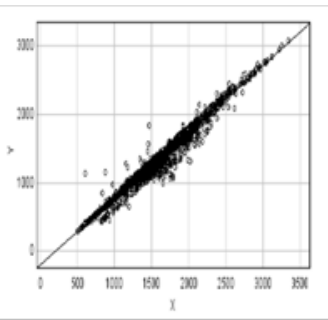

Detected Correspondence in closed condition

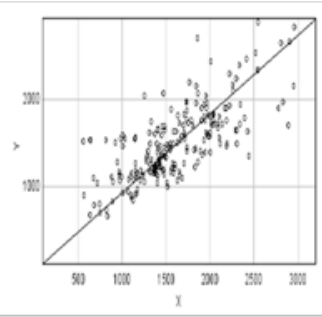

Detected Correspondence in open condition

Figure 2. Example for correspondences between reconstructed values in a VOI scan and a full scan: A linear correction function offers a good fit to correct for the mismatch. In closed condition, the patch matching delivers similar observations. If the scanned patient is excluded from the database, many outliers cause considerable noise in the matching process.

\section{RESULTS}

Due to the high spatial resolution and the sharp reconstruction kernel the average noise of the reconstructed images was about $140 \mathrm{HU}$ in homogenous areas. This noise posed a challenge to the parameter estimation process. In preliminary experiments, we found that parameter estimation based on least-squares methods did not lead to robust results. Details on this will be presented in the full paper.

Figure 2 gives an example for the quality of the matching process. For the estimation of the reference case, many correspondences are found to compute the correction parameters. Also in the closed condition, many matching feature vectors are extracted and good correspondences are found. Only for the open condition case, the correspondences contained a lot of noise. Thus, we expected a degraded performance in the open condition case.

Table 1 gives an overview of the estimated parameters in the different evaluation conditions. On average, the truncation correction yielded an offset of $133 \mathrm{HU}$. The slope of the transform was steady at an average of almost 1 . In closed condition, we could estimate the correction parameters very closely. The average error of the offset was only 9 HU. In case of the open condition, we still could achieve and error of only $23 \mathrm{HU}$ for the offset.

Table 2 reports root mean square errors between the full scan reconstruction and the VOI reconstruction. Before the correction the average error was $284 \mathrm{HU}$ which is about two times the noise level of the reconstructions. After correction the error was reduced to $147 \mathrm{HU}$ and $146 \mathrm{HU}$ with the reference correction and the correction estimated from the closed condition experiment respectively. In open condition the error is slightly higher with $162 \mathrm{HU}$.

Table 1: Overview of the parameter estimation results. As no error can be computed for the reference data, we report their average in parentheses.

\begin{tabular}{lrcrrrrr}
\hline & \multicolumn{2}{c}{ Reference } & \multicolumn{2}{c}{\begin{tabular}{c} 
Closed \\
\multicolumn{2}{c}{ Condition }
\end{tabular}} & \multicolumn{2}{c}{$\begin{array}{c}\text { Open } \\
\text { Condition }\end{array}$} \\
\hline Parameter & \multicolumn{1}{c}{$\mathrm{a}$} & \multicolumn{1}{c}{$\mathrm{b}$} & $\mathrm{a}$ & \multicolumn{1}{c}{$\mathrm{b}$} & $\mathrm{a}$ & $\mathrm{b}$ \\
$\mathbf{1}$ & 0.98 & -163 & 0.97 & -155 & 0.96 & -153 \\
$\mathbf{2}$ & 1 & -159 & 0.96 & -188 & 0.92 & -102 \\
$\mathbf{3}$ & 0.98 & -121 & 0.97 & -122 & 0.98 & -165 \\
$\mathbf{4}$ & 0.98 & -107 & 0.96 & -120 & 0.92 & -100 \\
$\mathbf{5}$ & 0.98 & -104 & 0.96 & -99 & 0.94 & -109 \\
$\mathbf{6}$ & 0.99 & -143 & 0.97 & -144 & 0.92 & -128 \\
Avg. Error & $\mathbf{( 0 . 9 8 )}$ & $\mathbf{( - 1 3 3 )}$ & $\mathbf{0 . 0 2}$ & $\mathbf{9}$ & $\mathbf{0 . 0 5}$ & $\mathbf{2 3}$ \\
\hline & & & & & &
\end{tabular}

Table 2: Overview of the root mean square error (RMSE) between the ROI reconstructions and the full scan reconstructions.

\begin{tabular}{lrrrr}
\hline ROI RMSE & $\begin{array}{l}\text { No } \\
\text { correction }\end{array}$ & $\begin{array}{r}\text { Reference } \\
\text { correction }\end{array}$ & $\begin{array}{l}\text { Closed } \\
\text { condition }\end{array}$ & $\begin{array}{l}\text { Open } \\
\text { condition }\end{array}$ \\
\hline $\mathbf{1}$ & 341 & 151 & 144 & 278 \\
$\mathbf{2}$ & 307 & 170 & 151 & 136 \\
\hline $\mathbf{3}$ & 296 & 149 & 151 & 122 \\
$\mathbf{4}$ & 271 & 133 & 125 & 119 \\
$\mathbf{5}$ & 263 & 134 & 137 & 156 \\
$\mathbf{6}$ & 291 & 146 & 171 & 159 \\
\hline Avg. Error & $\mathbf{2 8 4}$ & $\mathbf{1 4 7}$ & $\mathbf{1 4 6}$ & $\mathbf{1 6 2}$ \\
\hline
\end{tabular}




\section{SUMMARY}

The proposed Atlas-based linear VOI (ABL-VOI) image correction method is able to recover information that was lost in the truncation correction process. In our experiment, we were able to correct the images up to the noise level of the reconstructed images. In closed condition, the offset error was reduced to $9 \mathrm{HU}$ in open condition to $23 \mathrm{HU}$. In this manner angiographic VOI scans can be augmented with additional information for interventional use.

\section{ACKNOWLEDGEMENTS}

This work was supported by Siemens AG, Healthcare Sector. This work was never submitted, published, or presented before. The concepts and information presented in this paper are based on research and are not commercially available.

\section{REFERENCES}

[1] Dennerlein F, Maier A, "Region-Of-Interest Reconstruction on medical C-arms with the ATRACT Algorithm," in Proceedings SPIE 8313, San Diego, California, USA, 2012, p. 83131B.

[2] Fahrig R, Dixon R, Payne T, Morin RL, Ganguly A, Strobel N, "Dose and image quality for a cone-beam C-arm CT system," Medical Physics, vol. 33, no. 12, pp. 4541-50, 2006.

[3] Kolditz D, Kyriakou Y, Kalender WA, "Volume-of-interest (VOI) imaging in C-arm flat-detector CT for high image quality at reduced dose," Medical Physics, vol. 37, no. 6, pp. 2719-2730, 2010.

[4] Kudo H, Courdurier M, Noo F, Defrise M, "Tiny a priori knowledge solves the interior problem in computed tomography," Physics in medicine and biology, vol. 53, no. 9, pp. 2207-2231, 2008.

[5] Ohnesorge B, Flohr T, Schwarz K, Heiken JP, Bae KT, "Efficient correction for CT image artifacts caused by objects extending outside the scan field of view.," Medical Physics, vol. 27, no. 1, pp. 39-46, 2000.

[6] Hsieh J, Chao E. Thibault J, Grekowicz B, Horst A, McOlash S, "A novel reconstruction algorithm to extend the CT scan fieldof-view," Medical Physics, vol. 31, no. 9, pp. 2385-2391, 2004.

[7] Maier A, Scholz B, Dennerlein F, "Optimization-based Extrapolation for Truncation Correction," in 2nd CT Meeting, Salt Lake City, UT, USA, 2012, pp. 390-394.

[8] Xia Y, Maier A, Dennerlein F, Hofmann HG, Hornegger J, "Efficient 2D Filtering for Cone-Beam VOI Reconstruction," in 2012 IEEE MIC, Anaheim, CA, USA, 2012, p. to appear.

[9] Maier A, Wigström L, Hofmann HG, Hornegger J, Zhu L, Strobel N, Fahrig R, "Three-dimensional Anisotropic Adaptive Filtering of Projection Data for Noise Reduction in Cone Beam CT," Medical Physics, vol. 38, no. 11, pp. 5896-5909, 2011.

[10] Fahrig R, Fox AJ, Lownie S, Holdsworth DW, "Use of a C-arm system to generate true three-dimensional computed rotational angiograms: preliminary in vitro and in vivo results," American Journal of Neuroradiology, vol. 18, no. 8, pp. 1507-14, 1997.

[11] Maier A, Choi JH, Keil A, Niebler C, Sarmiento M, Fieselmann A, Gold G, Delp S, Fahrig R, "Analysis of Vertical and Horizontal Circular C-Arm Trajectories," in Proceedings SPIE 7961, Lake Buena Vista, Florida, USA, 2011, p. 796123.

[12] Bay H, Ess A, Tuytelaars T, Van Gool L, "Speeded-up robust Features (SURF)," Computer Vision and Image Understanding, vol. 110, pp. 346-359, 2008.

[13] Pearson K, "On lines and planes of closest fit to systems of points in space," Philosophical Mag., vol. 2, no. 6, pp. 559-572, 1901.

[14] Fischler MA, Bolles RC, "Random sample consensus: A paradigm for model fitting with applications to image analysis and automated cartography," Communications of the ACM, vol. 24, no. 6, pp. 381-395, 1981.

[15] Bögel M, Maier A, Hofmann HG, Hornegger J, Fahrig R, "Diaphragm Tracking for Respiratory Motion Compensated Cardiac CArm CT," in Proc. CT Meeting, Salt Lake City, UT, USA, 2012, pp. 13-16.

[16] Bögel M, Maier A, Hofmann HG, Hornegger J, Fahrig R, "Diaphragm Tracking in Cardiac C-Arm Projection Data," in Proc. BVM, Berlin, Germany, 2012, pp. 33-38. 